\title{
Three-Dimensional Echo Planar Imaging with Controlled Aliasing: A Sequence for High Temporal Resolution Functional MRI
}

\author{
Mayur Narsude, ${ }^{1,2}$ Daniel Gallichan, ${ }^{1}$ Wietske van der Zwaag, ${ }^{3}$ Rolf Gruetter, ${ }^{1,2,3,4}$ \\ and José P. Marques ${ }^{3,5}$ *
}

Purpose: In this work, we combine three-dimensional echo planar imaging (3D-EPI) with controlled aliasing to substantially increase temporal resolution in whole-brain functional MRI (fMRI) while minimizing geometry-factor (g-factor) losses.

Theory and Methods: The study was performed on a 7 Tesla scanner equipped with a 32-channel receive coil. The proposed 3D-EPI-CAIPI sequence was evaluated for: (i) image quality, compared with a conventionally undersampled parallel imaging acquisition; (ii) temporal resolution, the ability to sample and remove physiological signal fluctuations from the fMRI signal of interest and (iii) the ability to distinguish small changes in hemodynamic responses in an event-related fMRI experiment.

Results: Whole-brain fMRI data with a voxel size of $2 \times 2 \times$ $2 \mathrm{~mm}^{3}$ and temporal resolution of 371 ms could be acquired with acceptable image quality. Ten-fold parallel imaging accelerated 3D-EPI-CAIPI data were shown to lower the maximum g-factor losses up to $62 \%$ with respect to a 10 -fold accelerated 3D-EPI dataset. FMRI with $400 \mathrm{~ms}$ temporal resolution allowed the detection of time-to-peak variations in functional responses due to multisensory facilitation in temporal, occipital and frontal cortices.

Conclusion: 3D-EPI-CAIPI allows increased temporal resolution that enables better characterization of physiological noise, thus improving sensitivity to signal changes of interest. Furthermore, subtle changes in hemodynamic response dynamics can be studied in shorter scan times by avoiding the need for jittering. Magn Reson Med 75:2350-2361, 2016. ( 2015 Wiley Periodicals, Inc.

Key words: controlled aliasing; 3D EPI; functional MRI; physiological noise; parallel imaging; CAIPIRINHA

\footnotetext{
${ }^{1}$ Laboratory for Functional and Metabolic Imaging, Ecole Polytechnique Fédérale de Lausanne, Lausanne, Switzerland.

${ }^{2}$ Department of Radiology, University of Lausanne, Lausanne, Switzerland. ${ }^{3}$ Centre d'Imagerie BioMédicale, Ecole Polytechnique Fédérale de Lausanne, Lausanne, Switzerland.

${ }^{4}$ Department of Radiology, University of Geneva, Geneva, Switzerland.

${ }^{5}$ Donders Institute for Brain, Cognition and Behaviour, Radboud University, Nijmegen, The Netherlands

Grant sponsor: the Centre d'Imagerie BioMédicale (CIBM) of the University of Lausanne (UNIL); Grant sponsor: the Swiss Federal Institute of Technology Lausanne (EPFL); Grant sponsor: the University of Geneva (UniGe); Grant sponsor: the Centre Hospitalier Universitaire Vaudois (CHUV); Grant sponsor: the Hôpitaux Universitaires de Genève (HUG); Grant sponsor: the Leenaards and Jeantet Foundations.

*Correspondence to: José P. Marques, Ph.D., Kapittelweg 29, 6525 EN Nijmegen. E-mail: j.marques@donders.ru.nl

Received 28 November 2014; revised 16 June 2015; accepted 16 June 2015

DOI 10.1002/mrm.25835

Published online 14 July 2015 in Wiley Online Library (wileyonlinelibrary.com)
}

\section{INTRODUCTION}

Echo planar imaging (1), EPI, is the main pulse sequence used for functional MRI (fMRI) (2-4) due to its ability to offer high sampling rates. Standard multislice two-dimensional (2D) -EPI acquires fMRI data at spatial (2-4 mm isotropic) and temporal $(2-3$ s) resolutions which are sufficient to detect the hemodynamic response to neuronal activations (5). Research from various groups has demonstrated that both functional connectivity based studies $(6,7)$ as well as task-driven fMRI $(8,9)$ benefit from improved statistics attainable with a higher sampling rate. In addition, higher sampling rates allow separation of physiological signal fluctuations and blood oxygenation-level dependent (BOLD) signal of interest $(7,10-12)$.

At ultrahigh magnetic field strengths ( $\geq 7$ Tesla [T]), due to the increased signal available and because of the increased susceptibility induced artefacts, there is both an opportunity and a need to acquire higher spatial resolution images. Increasing the spatial resolution of multislice 2D-EPI suggests an increase not only in the echo train length (ETL), but also in the number of slices needed to achieve the same volume coverage, thereby increasing the minimum repetition time (TR) achievable for whole-brain coverage to a point where it becomes comparable to the hemodynamic response time ( $\sim 10-12 \mathrm{~s})$.

Recently, multiband (13-15) excitations have been proposed to accelerate the acquisition of $2 \mathrm{D}$ multislice EPI with successive slice shifting in the phase-encoding direction being used to reduce the g-factor penalty associated with the unfolding (14-17). Such approaches have shown their potential to provide sub-second temporal resolution in fMRI. The main penalty of the multiband excitation approach, especially at high fields, is the peak power of multiband pulses (18), although various methods have been developed to address this limitation (19-23).

Echo volume imaging (EVI) or single shot 3D-EPI $(9,24,25)$ is a technique where a thick slab is excited, as opposed to thin slices in the case of multislice 2D-EPI, and the full 3D volume is encoded during readout. 3D singleshot acquisitions require long echo trains resulting in compromises in the achievable echo time (TE) and spatial resolution and/or very high demands on the gradient hardware (9). Use of multislab echo volume imaging reduces the length of the echo train length and hence the strain on the gradients (12), however, it reduces the ability to use parallel imaging acceleration in the partition-encoding direction due to the reduced field of view (FOV). A more established strategy to overcome the limitations of EVI is 
the acquisition of the $3 \mathrm{D}$ volume in several shots (26-29) known as 3D-EPI. In this case, the time needed to acquire one entire $3 \mathrm{D}$ k-space $\left(\mathrm{TR}_{\text {volume }}\right)$ is $\mathrm{TR}_{\text {segment }}$ times the number of segments, where $\mathrm{TR}_{\text {segment }}$ is the time between successive excitations of the tissue volume. One or two k-space planes are typically acquired per excitation (30), leading to an ETL comparable to those of standard 2D-EPI. 3D-EPI provides multiple benefits over a multislice 2D-EPI approach, including a higher sensitivity per unit scan time $(31,32)$, the absence of a spin-history artifact, and the possibility of straightforward application of parallel imaging technique in both phase-encoding dimensions (28).

3D-EPI is more sensitive to physiological noise than its 2D counterpart $(10,11)$. Recent work has shown that the lower the number of segments used to form the 3D-EPI dataset, the higher is the maximum achievable temporal signal-to-noise ratio (tSNR) (29). A higher acceleration factor for partially parallel acquisition (PPA) in the partition-encoding direction results in a reduced number of segments used for volume acquisition, thus improving tSNR when operating in the physiological-noise dominated regime (29). As in all undersampled acquisitions, maximum acceleration is limited by the g-factor. Controlled aliasing in volumetric data (2D-CAIPIRINHA) has been proposed (33) as a means to reduce the noise amplification associated with parallel imaging reconstruction.

In this study, periodic gradient blips are played in the partition-encoding direction along with conventional phase-encoding blips in a segmented 3D-EPI sequenc to cover the k-space with a 2D-CAIPIRINHA pattern. We refer to this combination of segmented 3D-EPI and 2DCAIPIRINHA as 3D-EPI-CAIPI, which was recently presented in abstract form (34). Image quality assessment for high acceleration factors along the partition-encoding direction (hereafter referred to as $R_{Z}$ ) was performed by means of comparisons of 3D-EPI-CAIPI with highly accelerated standard 3D-EPI. Furthermore, the benefit of higher sampling rate acquisitions for resting state fMRI (rs-fMRI) following removal of physiological signal fluctuations from functional datasets was evaluated by acquiring 3D-EPI-CAIPI datasets at three different sampling rates. In the third experiment, 3DEPI-CAIPI with $400 \mathrm{~ms}$ temporal resolution and $2 \mathrm{~mm}$ nominal spatial resolution was used to demonstrate its ability to detect multisensory facilitation in the primary visual and auditory cortices, which has previously been demonstrated using a long TR, highly jittered fMRI acquisition (35).

\section{THEORY}

CAIPIRINHA k-space sampling patterns can readily be implemented into a segmented 3D-EPI sequence. Figure 1a shows the standard 3D-EPI sequence diagram and Figures $1 \mathrm{c}-\mathrm{d}$ show the modifications in the slice-encoding gradient to modify a 3D-EPI sequence into 3D-EPI-CAIPI. Resulting k-space sampling patterns (Figs. 1b-d), are shown for three possible trajectories to cover the k-space with the same net acceleration $R=10$, with $R_{z}=5$ (to ensure a five-fold increase in temporal resolution and five-fold reduction in the number of excitations per volume) and $R_{y}=2$ (to ensure reduced image distortions and to achieve an optimal TE). Each sampling point in the yz-plane is a k-space line along the read direction during the EPI readout with the readout along the $\mathrm{x}$ direction. The solid circles in the sampling patterns (Figures $1 \mathrm{~b}-\mathrm{d}$, right-hand side) indicate k-space sampling points in the yz-plane that are acquired, whereas empty squares indicate un-acquired k-space sampling points. 2D-CAIPIRINHA trajectories are characterized by the $\Delta$ term (33), which indicates the shift used along the partition-encoding direction while acquiring each successive $\mathrm{k}$-space point in the primary phase-encoding direction. Standard parallel imaging undersampling strategies as used in 3D-EPI correspond to $\Delta=0$ (Fig. 1b). When a non-zero $\Delta$ is used (Figs. 1c-d), gradient blips along the partition-encoding direction are played together with the primary phase-encoding blips to shift the successive k-space positions in the partitionencoding direction by $\Delta$. When the cumulative k-space shift along the partition-encoding direction is greater than or equal to $\mathrm{Rz}$, the partition-encoding gradient moment is rewound and the process is repeated. With $\Delta=0$, i.e., standard undersampling, the resulting aliasing artifacts appear only in the undersampled direction. Whereas when a non-zero $\Delta$ is used, the notion of inplane and through-plane acceleration diffused, resulting in more scattered aliasing patterns, as described in Breuer et al (33), that make a more effective use of the available FOV. Another notion that can be useful when interpreting a new k-space sampling pattern is that the distance between a nonsampled point and their closest source of information should be minimized. The reduction of this distance facilitates the kernel fitting (although for a clearer picture the shape of the coils and their sensitive field also plays an important role at reweighting the distances in $\mathrm{k}_{\mathrm{y}}$ and $\mathrm{k}_{\mathrm{z}}$ ). It is worth noting that the k-space sampling trajectories shown in Figures $1 \mathrm{~b}-\mathrm{d}$ for 3D-EPI-CAIPI are identical to the trajectories used in simultaneous multislice blipped 2D-EPI.

\section{METHODS}

All experiments were conducted according to procedures approved by the institutional review board and all participants provided written informed consent before the experiments. A 7T/680mm head-only scanner (Magnetom 7T, Siemens, Erlangen, Germany) equipped with a head gradient insert $(80 \mathrm{mT} / \mathrm{m}$ maximum gradient strength, $333 \mathrm{~T} / \mathrm{m} / \mathrm{s}$ maximum slew-rate) and a 32-channel RF-head coil (Nova Medical Inc., MA) were used.

Frequency-selective fat saturation pulses were applied during EPI acquisitions before each excitation pulse to reduce the chemical shift artifacts. The Ernst angle was used throughout the study assuming an average $\mathrm{T}_{1}$ value of $1800 \mathrm{~ms}$ and the $\mathrm{TR}_{\text {segment }}$ of each acquisition. 3D-EPI-CAIPI image reconstructions were performed on a standalone computer equipped with MATLAB (R2012a, Mathworks Inc.) using a 2D-GRAPPA reconstruction method (36).

The GRAPPA reconstruction, for a given acceleration only along the $y$-and $z$-direction, can be represented as 

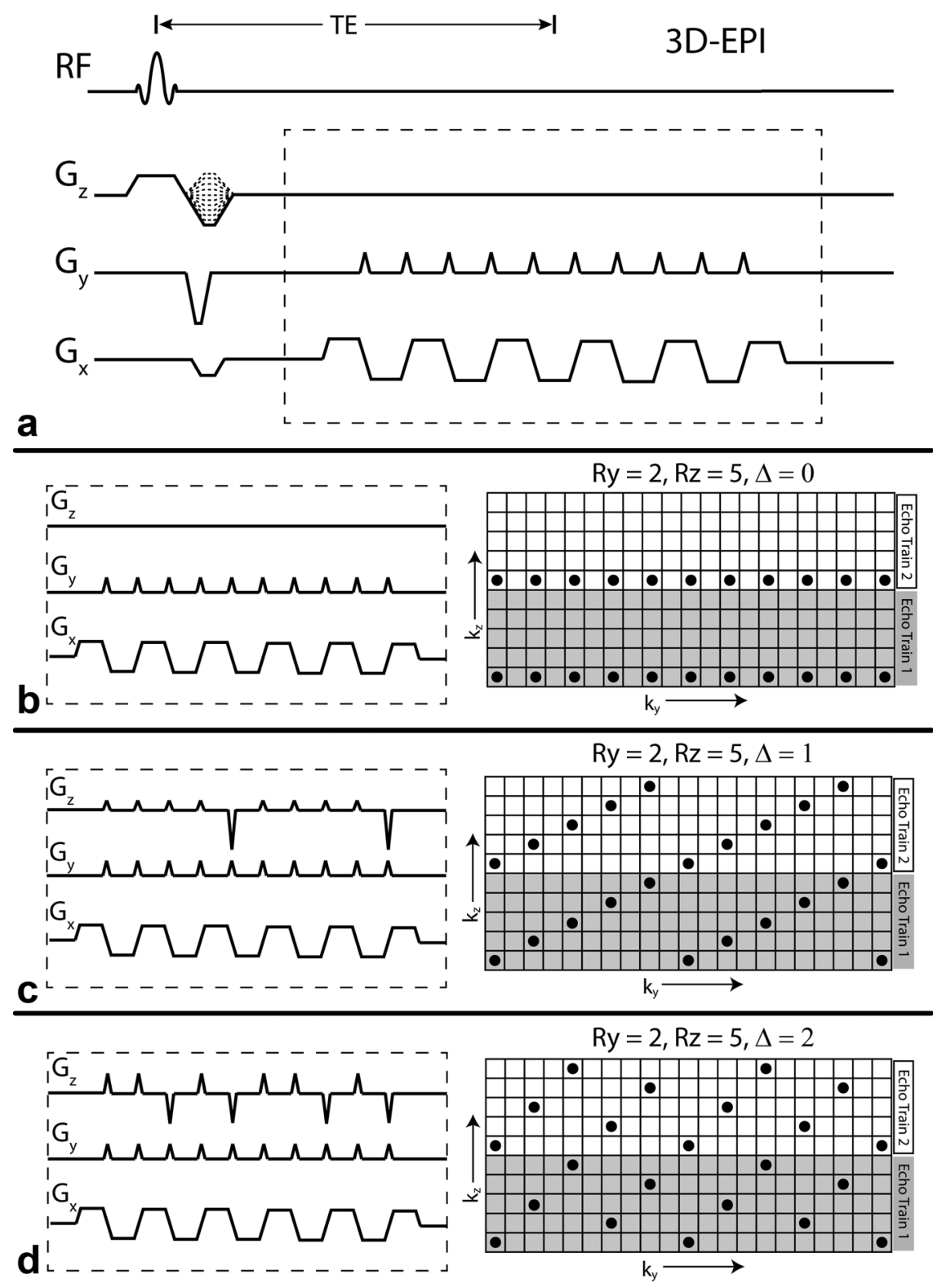

FIG. 1. a: Pulse sequence diagram for segmented 3D-EPI pulse sequence. The dotted box outlines partition-, primary phase-, and read-gradients $(z, y$, and $x$ axes, respectively) which are modified to shift successive k-space samples in the partition-encoding direction to achieve controlled aliasing. Gradient waveforms and resulting k-space sampling patterns for total acceleration $R=R_{y} \times R_{z}=2 \times 5$ are shown for $\Delta=0$ (b), $\Delta=1$ (c), and $\Delta=2$ (d). b: $\left(R_{y} \times R_{z}=2 \times 5, \Delta=0\right), 2 / 9$ points that have to be computed have a minimum distance to a source point of $2.24 \delta \mathrm{k}$ (where $\delta k$ is the spacing between successive k-space coordinates). In the case of $\Delta=1$, this distance only happens for $1 / 9$ points, while for and $\Delta=2$ there are $2 / 9$ points at a distance of $2 \mathrm{\delta k}$.

$$
\begin{aligned}
& S_{j}\left(x, k_{y}+\delta k_{y},(t), k_{z}+\delta k_{z}(t)\right)=\sum_{l=1}^{N C o i l s} \sum_{s=1}^{N S} w_{x, j, t}(l, s) \\
& \quad \times S_{l}\left(x, k_{y}+\Delta k_{y}(s), k_{z}+\Delta k_{z}(s)\right)
\end{aligned}
$$

where the un-acquired signal of coil $j$ in the hybrid space, $S_{j}\left(x, k_{y}, k_{z}\right)$, on the left-hand side of Eq. [1] can be obtained by a linear combination of the acquired hybrid space signals by the different receivers. In this formalism, all the $\left(\mathrm{k}_{\mathrm{y}}, \mathrm{k}_{\mathrm{z}}\right) \mathrm{k}$-space data are acquired phase encoding coordinates, $\left(\delta \mathrm{k}_{\mathrm{y}}(\mathrm{t}), \delta \mathrm{k}_{\mathrm{z}}(\mathrm{t})\right)$ and $\left(\Delta \mathrm{k}_{\mathrm{y}}(\mathrm{s}), \Delta \mathrm{k}_{\mathrm{z}}(\mathrm{s})\right)$ are the GRAPPA kernel coordinates corresponding to target and source points, respectively (see Supporting Figure S1, which is available online). Finally, $w_{x, j, t}$ are the GRAPPA weights for coil $j$ and target point $t$ at the position $\mathrm{x}$ in the readout direction, NCoils is the total number of receiver coils and $N S$ is the total number of source points in the kernel. This process has to be repeated for all target coils, target points in the GRAPPA kernel and $\mathrm{x}$ - positions. The kernel is then shifted to fill a new k-space region. The above formulation of GRAPPA can be simplified as a matrix equation $\mathbf{b}$

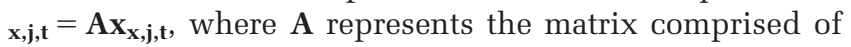
the acquired data, $\mathbf{b}$ denotes the vector of the missing data, and $\mathbf{x}$ represents the coefficient vector (for the sake of notation simplicity the indexes $\mathrm{x}, \mathrm{j}, \mathrm{t}$ will be discarded from now on).

In GRAPPA, autocalibration data are acquired and used as the vector $\mathbf{b}$ to estimate the coefficient vector $\mathbf{x}$. The least-squares method is commonly used to calculate the coefficients:

$$
\hat{X}=\min _{x}\|\mathbf{b}-\mathbf{A x}\|^{2}
$$

which can be solved by matrix inversion, $\mathbf{x}=\mathbf{A}^{-1} \mathbf{b}$. When the inversion of the matrix $\mathbf{A}$ is ill-conditioned due to the high acceleration factors and/or the presence of various coils with low SNR, noise can be greatly amplified in the estimated coefficients. Regularization 
methods $(37,38)$ have been used to condition the calculation of GRAPPA weights by

$$
\hat{\mathbf{X}}=\min _{x}\|\mathbf{b}-\mathbf{A} \mathbf{x}\|^{2}+\lambda R(x),
$$

where $R(x)$ is a regularization function $\left(R(x)=\|x\|_{2}\right.$ in the case of the Tikhonov regularization used in this manuscript) and $\lambda$ is the regularization parameter. The amount of regularization can be adjusted to reduce noise at the expense of additional uncorrected aliasing.

For reconstruction of 3D-EPI-CAIPI images, before the application of GRAPPA, the acquired data were Fourier transformed along the readout direction and a linear phase correction algorithm was applied (39). Subsequently, each $\mathrm{k}_{\mathrm{y}}-\mathrm{k}_{\mathrm{z}}$ plane (at a fixed $\mathrm{x}$-position) was treated as a 2D k-space plane and used as input for GRAPPA reconstruction algorithm. The GRAPPA autocalibration signal was measured before the undersampled acquisition using the same 3D-EPI readout train as for the main sequence. Care was taken to ensure that the segmented EPI trains corresponding to the primary phase-encoding lines were acquired in the inner loop while the secondary phase-encoding loop was kept as the outer loop. Note that, as opposed to multiband 2D-EPI, in 3D EPI, the steady state is not altered by such an ordering loop, but the same advantages can be found: reduction of reconstruction artifacts associated with large physiological state variations in neighboring k-space data of the ACS data (40). The undersampled (accelerated) data were then acquired using sampling patterns mentioned in the theory section. G-factor maps were calculated using the general formulation for quantitative g-factor calculations in GRAPPA reconstructions (41).

$\mathrm{B}_{1}$ maps were acquired using the Sa2RAGE sequence (42) to ensure correct RF calibration for all the experiments performed. The average $B_{1}$ value over the whole-brain was assessed to calibrate the reference voltage at the start of each individual scan session. Anatomical images were acquired with the MP2RAGE sequence (43) with $\mathrm{TR}_{\mathrm{MP} 2 \mathrm{RAGE}}=6.0 \mathrm{~s}$, $\mathrm{TE}=3.03 \mathrm{~ms}, \quad \mathrm{TI}_{1} / \mathrm{TI}_{2}=0.8 / 2.7 \mathrm{~s}, \quad \alpha_{1} / \alpha_{2}=7^{\circ} / 5^{\circ}$, matrix size $=320 \times 320 \times 256,0.6 \times 0.6 \times 0.6 \mathrm{~mm}^{3}$ isotropic voxel size, slice encode $=$ left-right, phase encode $=$ anteriorposterior, PPA (partially parallel acceleration factor) $=3 \times$ $1, \mathrm{PF}_{\mathrm{y}}$ (partial-Fourier factor in the primary phase-encoding direction) $=6 / 8$ and $\mathrm{PF}_{\mathrm{z}}$ (partial Fourier factor in the partition-encoding direction) $=6 / 8$.

\section{Image Quality Assessment}

Image quality was evaluated in $1.0 \mathrm{~mm}$ isotropic wholebrain images acquired from a single subject with 3D-EPICAIPI with different net accelerations and $\Delta$ values. The acquisition parameters used were: $\mathrm{TR}_{\text {segment }} / \mathrm{TE}=67 / 26$ ms, flip angle $(\mathrm{FA})=14^{\circ}$, readout bandwidth $(\mathrm{rBW})=1662 \mathrm{~Hz} /$ Pixel, matrix size $=188 \times 188 \times 120$, reference data $=188 \times 48 \times 48$, GRAPPA kernel size $=3 \times 3, \mathrm{PPA}_{\mathrm{y}}=2$ and $\mathrm{PF}_{\mathrm{y}}=7 / 8 . \mathrm{PPA}_{\mathrm{z}}$ varied from 3 to 5. With $\mathrm{PPA}_{\mathrm{z}}=5$, an acquisition with $\mathrm{PF}_{\mathrm{z}}=6 / 8$ was performed to achieve a total data reduction factor of $\mathrm{R}_{\mathrm{z}}=6.66$ in the $\mathrm{z}$-direction, and $\mathrm{R}=15.24$ overall, followed by homodyne reconstruction $(44,45)$. The partition- encoding direction was set to superior-inferior while the primary phase-encoding direction was set right-left.

\section{Physiological Noise Characterization}

To investigate the ability of shorter $\mathrm{TR}_{\text {volume }}$ acquisitions to better characterize respiratory and cardiac induced signal fluctuations, MR data were acquired using 3D-EPICAIPI with three different volume TRs from three subjects (two males, average age $=25.3$ years) with simultaneous monitoring of the respiratory and cardiac cycles. Common protocol parameters used for these runs were: $\mathrm{TR}_{\text {segment }} / \mathrm{TE}=53 / 26 \mathrm{~ms}, \mathrm{FA}=13^{\circ}$, matrix size $=106 \times$ 106, $2 \mathrm{~mm}$ isometric voxel size, $\mathrm{PPA}_{\mathrm{y}}=1, \mathrm{rBW}=3144$ Hz/Pixel. Differences in protocol parameters used to achieve different volume TRs were:

1. $\mathrm{TR}_{\text {volume }}=3.180 \mathrm{~s}:$ volumes $=94, \mathrm{~N}_{\mathrm{par}}=60, \mathrm{~N}_{\mathrm{seg}}=60$, $\mathrm{PPA}_{\mathrm{z}}=1$

2. $\mathrm{TR}_{\text {volume }}=1.060 \mathrm{~s}:$ volumes $=283, \mathrm{~N}_{\mathrm{par}}=60, \mathrm{~N}_{\mathrm{seg}}=20$, $\mathrm{PPA}_{\mathrm{z}}=3, \Delta=1$, reference data $=106 \times 48 \times 39$, kernel size $=3 \times 3$

3. $\mathrm{TR}_{\mathrm{volume}}=0.371 \mathrm{~s}$ : volumes $=808, \mathrm{~N}_{\mathrm{par}}=60, \mathrm{~N}_{\mathrm{seg}}=8$, $\mathrm{PPA}_{\mathrm{z}}=6, \Delta=2$, reference data $=106 \times 48 \times 39$, kernel size $=3 \times 3, \mathrm{PF}_{\mathrm{z}}=6 / 8$

4. $\mathrm{TR}_{\mathrm{volume}}=1.060 \mathrm{~s}$ : volumes $=283, \mathrm{~N}_{\mathrm{par}}=20, \mathrm{~N}_{\mathrm{seg}}=20$, $\mathrm{PPA}_{\mathrm{z}}=1$, reference data $=106 \times 48 \times 20$, kernel size $=3 \times 3$

5. $\mathrm{TR}_{\mathrm{volume}}=0.371 \mathrm{~s}$ : volumes $=808, \mathrm{~N}_{\mathrm{par}}=8, \mathrm{~N}_{\mathrm{seg}}=8$, $\mathrm{PPA}_{\mathrm{z}}=1$, reference data $=106 \times 48 \times 8$, kernel size $=3 \times 3$

Two runs at each sampling rate were performed and their order was randomized. Power spectrum analysis was performed in MATLAB on both physiological data and MR signals. All datasets were temporally low-pass filtered with a cut-off frequency of $0.14 \mathrm{~Hz}$ using a Butterworth filter to filter out signal fluctuations due to physiological processes such as respiration and cardiac pulsation. All datasets were coregistered with standard T1-weighted Talairach brain template in Statistical Parametric Mapping (SPM, http://www.fil.ion.ucl.ac.uk/ $\mathrm{spm} /$ ) software. Maps of tSNR per unit scan time, defined as the mean divided by the standard deviation along the time dimension divided by the square root of the $\mathrm{TR}_{\mathrm{volume}}$ used for the acquisition, were created preand post-lowpass filtering for whole-brain, white matter, and gray matter regions separately. Note that this definition makes a fair comparison between the ability of sequences with different sampling rates to detect changes in the BOLD signal in a fixed amount of sampling time. White and gray matter masks were created using SPM segmentation and this masks were used to compute the tSNR in different white and gray matter regions for the three subjects.

Protocols 4 and 5 (partial brain acquisitions) were introduced to control whether the improvement in tSNR per unit of time observed was an artificial effect introduced by the CAIPI reconstruction or if it was truly related with the reduction of $\mathrm{TR}_{\mathrm{volume}}$.

Whole-brain datasets from a single subject (based on protocols 1, 2, and 3) were motion corrected using MCFLIRT. The data were then low-pass filtered (as in 
the previous paragraph) and finally, the $1.060 \mathrm{~s}$ and $0.371 \mathrm{~s}$ datasets were temporally down-sampled using cubic spline interpolation to create an effective TRvolume of $3.180 \mathrm{~s}$ producing 94 volumes each. Temporal drifts were removed using a high-pass filter with a cutoff at $100 \mathrm{~s}$. Datasets were aligned to the high resolution MP2RAGE anatomical images using FLIRT (46-49). Spatial smoothing of $3 \mathrm{~mm}$ full-width-half-maximum (FWHM) was applied. RSNs were identified using the MELODIC toolbox from FSL (http://www.fmrib.ox.ac.uk) which performs Independent Component Analysis (ICA) $(49,50)$. RSNs that consistently appeared at all the TRs were automatically identified by choosing the components with spatial correlation greater than 0.35 (after thresholding the component maps at $\mathrm{Z}=5$ ). Additional components were identified after visual inspection. Names for each RSN were assigned by means of visual comparison to published data $(22,51-54)$

\section{Changes in HRF Dynamics Due to Multisensory Integration}

To demonstrate the ability of 3D-EPI-CAIPI to detect multisensory facilitation in the primary visual and auditory cortices with short TR, three volunteers (two females, one male) were scanned using a protocol that used a stimulation paradigm closely mimicking that described in Martuzzi et al (35): volunteers were asked to report by means of a button-press the occurrence of either a visual flash $(\mathrm{V}, 150 \mathrm{~ms}$, yellow/black checkerboard), a sound presentation [A, $150 \mathrm{~ms}$, white noise burst delivered by means of Sensimetrics earplugs (Sensimetrics Corporation, MA)] or a simultaneous combination of the two (AV). Per 8-min run, 30 stimuli were presented using E-prime (Psychology Software Tools Inc., PA), which was also used to record response times. Stimuli were presented every 15-17 s.

Data acquisition was performed using the 3D-EPI-CAIPI acquisition with acquisition parameters similar to the $\mathrm{TR}_{\text {volume }} 0.371 \mathrm{~s}$ protocol used for the physiological noise characterization experiment, i.e.: $\mathrm{TR}_{\mathrm{volume}}=399 \mathrm{~ms}$ (seven segments, $\mathrm{TR}_{\text {segment }}=57 \mathrm{~ms}$ ), nominal spatial resolution $2 \times 2 \times 2 \mathrm{~mm}^{3}$, matrix size $106 \times 106 \times 60, \mathrm{TE}=26 \mathrm{~ms}$, $\mathrm{FA}=13^{\circ}, \mathrm{PPA}=1 \times 6, \Delta=2$, reference data $=106 \times 48 \times$ 39 , kernel size $=3 \times 3, \mathrm{PF}_{\mathrm{z}}=6 / 8, \mathrm{rBW}=2774 \mathrm{~Hz} / \mathrm{Px}$, slab was anterior-posterior commissure aligned with the primary phase-encoding direction right to left. Two functional runs were acquired, each consisting of 1215 volumes.

SPM8 (http://www.fil.ion.ucl.ac.uk/spm) was used for motion correction, smoothing (FWHM $3 \mathrm{~mm}$ ) and a generalized linear model analysis with regressors for A, V, and AV stimuli and the temporal derivatives thereof. Regions of interest (ROIs) of $\sim 500$ voxels were defined in the auditory, visual, and left motor cortices from the $\mathrm{A}>\mathrm{V}, \mathrm{V}>\mathrm{A}$, and f-test over $(\mathrm{A}, \mathrm{V})$ respectively. From each ROI, timecourses were extracted, temporally filtered to remove slow drifts and subsequently temporally reordered relative to the stimulus onset. A double gamma function (54) was fitted to the reordered timecourses to allow calculation of the time-to-peak.

\section{RESULTS}

\section{Image Quality Assessment}

Three orthogonal planes of $1 \mathrm{~mm}$ isotropic resolution images are shown along with their $1 / \mathrm{g}$ maps in Figure 2 for different $\mathrm{R}_{\mathrm{z}}$. For all the acquisitions, $\mathrm{R}_{\mathrm{y}}$ was kept constant at 2 whereas $R_{z}$ was varied and is indicated on the far left of each row along with resulting $\mathrm{TR}_{\text {volume }}$ for each acquisition. Standard sampling pattern images, i.e., $\Delta=0$, exhibited greater g-factor losses (Figure 2, first column) than those acquired with CAIPIRINHA sampling patterns, i.e., $\Delta>0$ (Figure 2, center and right column). The average $1 / \mathrm{g}$ values calculated over the whole-brain are reported at the top of each $1 / \mathrm{g}$ map panel.

The benefit of using CAIPIRINHA with 3D-EPI acquisitions was noticeable particularly for highly undersampled acquisitions. For PPA $=2 \times 5$ and $\Delta=0$, (Fig. 2I) most of the brain anatomy was not recognizable (especially in the central part of the brain) but this was progressively recovered with $\Delta=1$ (Fig. 2J) and $\Delta=2$ (Fig. 2K). The improvements in SNR were ascribed to the reduced g-factor. The whole-brain averaged $1 / \mathrm{g}$ values were 0.70 with $\Delta=0$ and 0.77 with $\Delta=2$, corresponding to a $10 \%$ improvement in average retained SNR value over the whole-brain. It should also be noted that the minimum $1 / \mathrm{g}$ value was 0.26 with $\Delta=0$, which increased to 0.42 with $\Delta=2$, an improvement of $\sim 62 \%$ in the worst-case retained SNR value. At lower acceleration, reconstruction improvements were not evident from simple visual inspection of the images. However, for the lowest acceleration of $2 \times 3$ (Figs. $2 \mathrm{~A}-\mathrm{B}$ ), average $1 / \mathrm{g}$ values increased from $0.90(\Delta=0)$ to $0.93(\Delta=1)$ with the use of CAIPIRINHA, an increase of $\sim 3 \%$. Figures $2 \mathrm{~L}$ and $2 \mathrm{M}$ show transverse and sagittal planes from Figures $2 \mathrm{~B}$ and $2 \mathrm{~K}$ respectively to help appreciate comparable image quality obtained even though the latter was obtained with a 2.2 -fold shorter $\mathrm{TR}_{\text {volume. }}$

\section{Physiological Noise Characterization}

To assess the improvement in physiological noise characterization at higher sampling rates, for each of the three different sampling rates, the power spectra of the physiological signals (respiration and cardiac signals) and the average power spectra of the whole-brain from one of the subjects during the fMRI time course were calculated and plotted together in Figure 3. With the lowest sampling rate used $\left(\mathrm{TR}_{\mathrm{volume}}=3.18 \mathrm{~s}\right.$ or $\left.0.31 \mathrm{~Hz}\right)$, both respiratory and cardiac peaks, which were observed at $\sim 0.28 \mathrm{~Hz}$ and $\sim 1.26 \mathrm{~Hz}$, respectively, could not be sampled adequately, since the highest frequency signal that could be sampled faithfully was $\sim 0.16 \mathrm{~Hz}$. As a result, aliased peaks of respiratory and cardiac signal fluctuations (at $\sim 0.12 \mathrm{~Hz}$ and $\sim 0.14 \mathrm{~Hz}$, respectively) are likely to interfere with the frequency range where the task-free BOLD signal is usually observed (i.e., $<0.1 \mathrm{~Hz}$ ) (55). A $\mathrm{TR}_{\mathrm{volume}}$ of $1.06 \mathrm{~s}$ critically samples a highest frequency of $0.47 \mathrm{~Hz}$ and hence could sample the respiratory peak well. However, in this subject, the cardiac peak was aliased at $\sim 0.32 \mathrm{~Hz}$, indicated by the arrow in Figure $3 \mathrm{~b}$. With $\mathrm{TR}_{\mathrm{volume}}$ of $0.37 \mathrm{~s}$, both the respiratory 


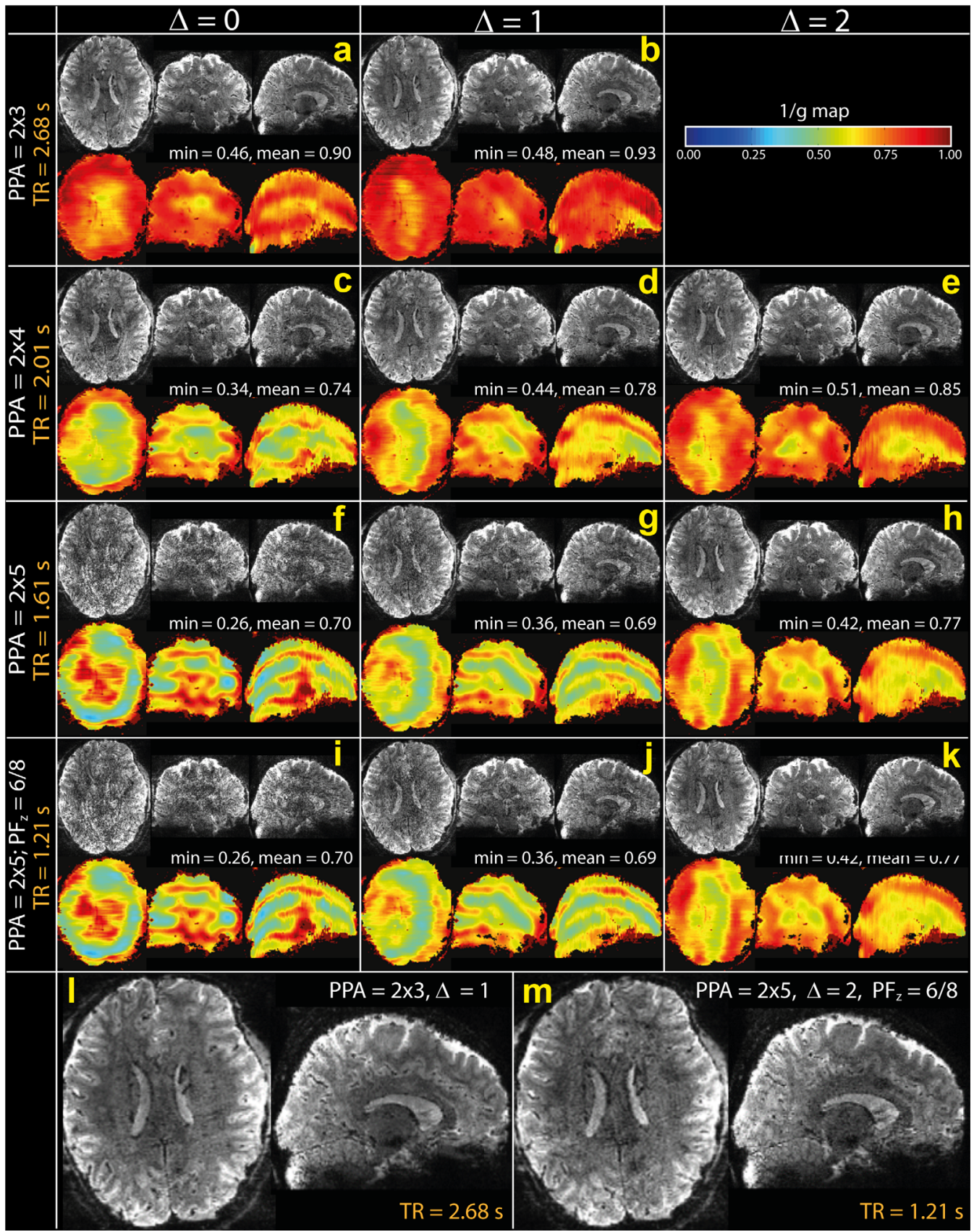

FIG. 2. A-M: Whole-brain echo planar images along with 1/g maps shown in orthogonal planes acquired with 3D-EPI-CAIPI for a single subject. Different parallel imaging acceleration factors used along with resulting volume TRs are indicated on the left of each image row. The left column shows the case of standard parallel imaging $(\Delta=0)$, the middle column shows controlled aliasing with $\Delta=1$ and the right column shows the $\Delta=2$ case. The averaged $1 / \mathrm{g}$ values are reported on the top right of each $1 / \mathrm{g}$ map. Panels (L) and (M) show enlarged transverse and sagittal planes from panels $(\mathrm{B})$ and $(\mathrm{K})$, respectively, for better visualization. All the images presented are single time-point images without further processing.

and cardiac peaks were below the Nyquist rate of $\sim 1.34$ $\mathrm{Hz}$ and hence well-sampled.

The insets in Figure 3 show orthogonal slices of the datasets acquired for power spectral analysis. As expected, the $1.06 \mathrm{~s}$ dataset (Fig. 3b) exhibited lower gfactor losses (average $1 / \mathrm{g}=0.97$ ) compared with the $0.371 \mathrm{~s}$ dataset (Figure 3c, average $1 / \mathrm{g}=0.84$ ). The image quality with both sampling rates was considered acceptable by visual inspection, and the eight-fold acquisition speed-up resulted in only $\sim 13 \%$ additional g-factor losses compared with three-fold acceleration, attributed to the 2D-CAIPIRINHA sampling scheme (which was also used for the three-fold accelerated data).

When examining tSNR/time maps averaged across subjects for all three sampling rates, no significant change in tSNR per unit time values was observed pre- and postlowpass filtering for the $3.18 \mathrm{~s}$ data, as expected (Figs. $4 \mathrm{~A}-\mathrm{F}, 5$ ), because neither of the physiological signals 
could be filtered out by means of low-pass filtering from this dataset. Both $1.06 \mathrm{~s}$ and $0.37 \mathrm{~s}$ datasets, which already exhibited higher tSNR per unit time values compared with $3.18 \mathrm{~s}$ dataset, could benefit further from the application of low-pass filtering. The whole-brain averaged tSNR per unit time values for the $0.37 \mathrm{~s}, 1.06 \mathrm{~s}$, and $3.18 \mathrm{~s}$ dataset before low-pass filtering were 46.5, 32.7, and 21.3, respectively. After low-pass filtering these values increased to $66.2,42.6$, and 21.5. This corresponds to an increase in tSNR per unit time of $42 \%$ and $30 \%$ for the datasets with a TR of 0.37 and 1.06, respectively. In the case of partial brain acquisitions (Figs. 4G,H,K,L), the values of tSNR obtained showed the same trend as when accelerating with CAIPI with the tSNR per unit of time increasing as the TR is reduced. The increase in tSNR per unit time due to low-pass filtering was observed to be $45 \%$ and $37 \%$ for $0.37 \mathrm{~s}$ and $1.06 \mathrm{~s}$ sampling rates, respectively, while again no significant change in tSNR per unit time was observed for the $3.18 \mathrm{~s}$ dataset after low-pass filtering.

In Figure 5, it can be seen that tSNR per unit time values showed significant disparities between white and gray matter (white matter always higher that gray matter), but were reproducible across subjects (see error bars). Different gray matter regions were analyzed to evaluate if the spatially varying reconstruction noise could locally reduce the temporal SNR per unit of time associated with TR reduction. All regions showed comparable benefits from the removal of frequency components associated with cardiac and respiratory fluctuations.

Figure 6 shows six representative RSNs identified with the three sampling rates used. The original volume TRs are indicated on the left of each panel. The networks were found to have higher statistical significance, to show larger activated areas and to have higher maximum z-score values (see Table 1) using faster sampling comparing (top to bottom) volume TRs $3.18 \mathrm{~s}, 1.06 \mathrm{~s}$, and $0.37 \mathrm{~s}$.

\section{Changes in HRF Dynamics Due to Multisensory Integration}

Multisensory facilitation in the changes in HRF dynamics study was evident from the recorded reaction times, with significant differences $(P<0.001)$ in reaction speed between audiovisual (AV) and both auditory (A) and visual (V) stimuli (Fig. 7a). Martuzzi et al reported longer reaction times for $\mathrm{A}$ than for $\mathrm{V}$ stimuli, but also noted that this differed per subject (35). An example activation map for a single subject shown overlaid on the MP2RAGE anatomical shows the spatial resolution was sufficient to localize well the BOLD signal changes to the gray matter (Fig. 7b), while yielding very high BOLD sensitivity; these maps were obtained from $A>V$ and $\mathrm{V}>\mathrm{A}$ contrasts with only 20 individual $150 \mu$ s events for each stimulus type.

ROI timecourses averaged over runs and subjects are shown in Figure 7 for the visual cortex (Fig. 7c), auditory cortex (Fig. 7d), and motor cortex (Fig. 7e). The doublegamma function described the BOLD responses well to the different stimuli. The time-to-peak after stimulus
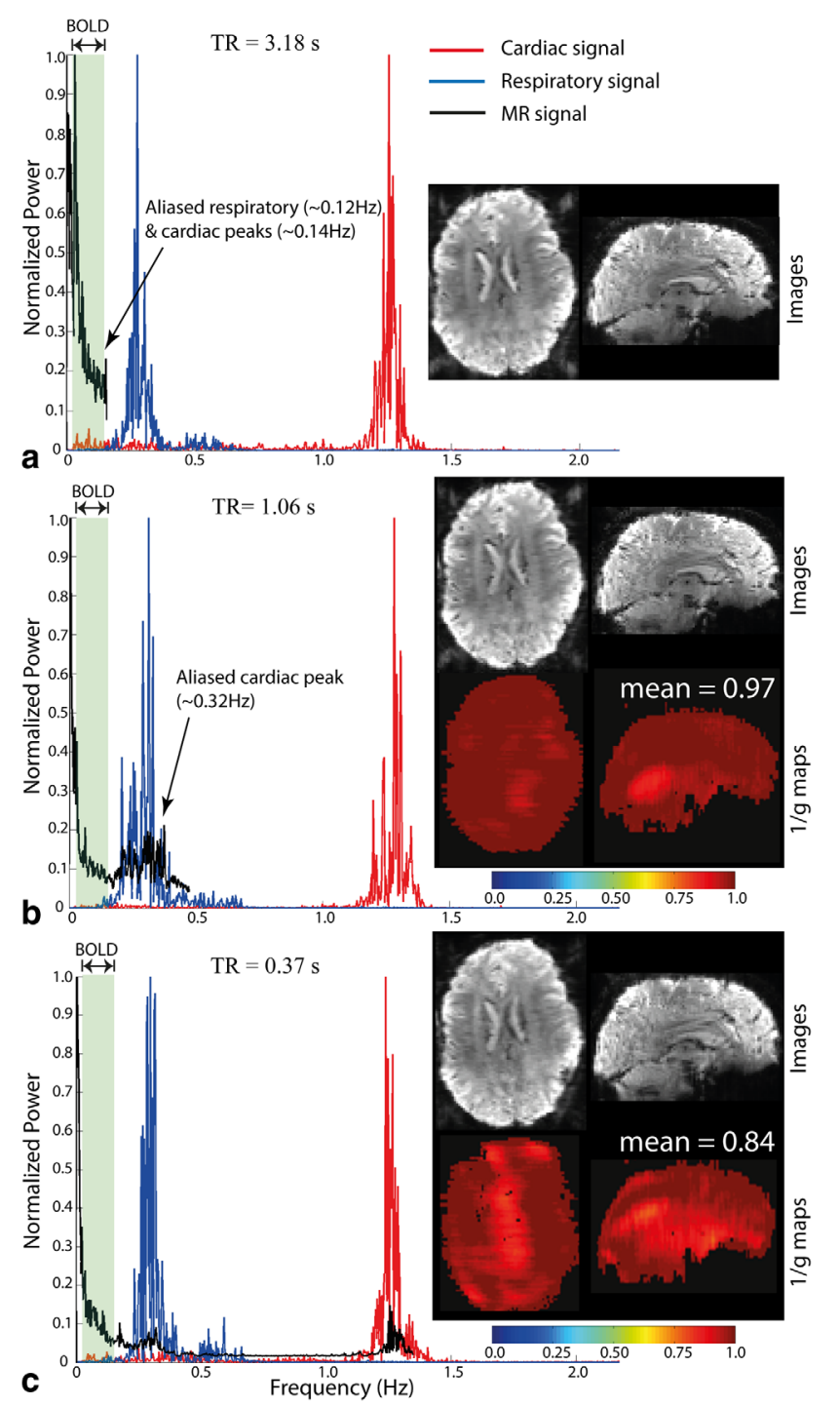

FIG. 3. Power spectra of the respiratory, cardiac, and MR data obtained with $\mathrm{TR}_{\text {volume }}$ of $3.18 \mathrm{~s}(\mathbf{a}), 1.06 \mathrm{~s}$ (b), and $0.37 \mathrm{~s}$ (c). BOLD signals are typically found in the $0.04-0.13 \mathrm{~Hz}$ frequency range shown by the green vertical band. Inset: Whole-brain images along with $1 / \mathrm{g}$ maps shown in orthogonal planes acquired at corresponding sampling rates (only images for $3.18 \mathrm{~s}$ dataset). The averaged $1 / \mathrm{g}$ values are also reported on the top right of each $1 / \mathrm{g}$ map. All the images presented are single time-point images without further processing.

onset for the fitted double gamma functions is given in Table 2. Positive responses for auditory stimuli were found in the visual cortex and vice versa, albeit significantly delayed compared with the cortex-own stimuli (Table 2), as well as much lower in amplitude, as was also reported in Martuzzi et al (35). As can be observed from the timecourses in Figure 7, as well as the times-topeak in Table 2, BOLD responses to the AV stimulus occurred measurably earlier than either of the singleinput stimuli, in agreement with (35). This effect was found in all three ROIs, i.e., AV stimuli were followed by earlier BOLD peaks in the occipital cortex than $\mathrm{V}$ stimuli, earlier BOLD peaks in the temporal cortex than 


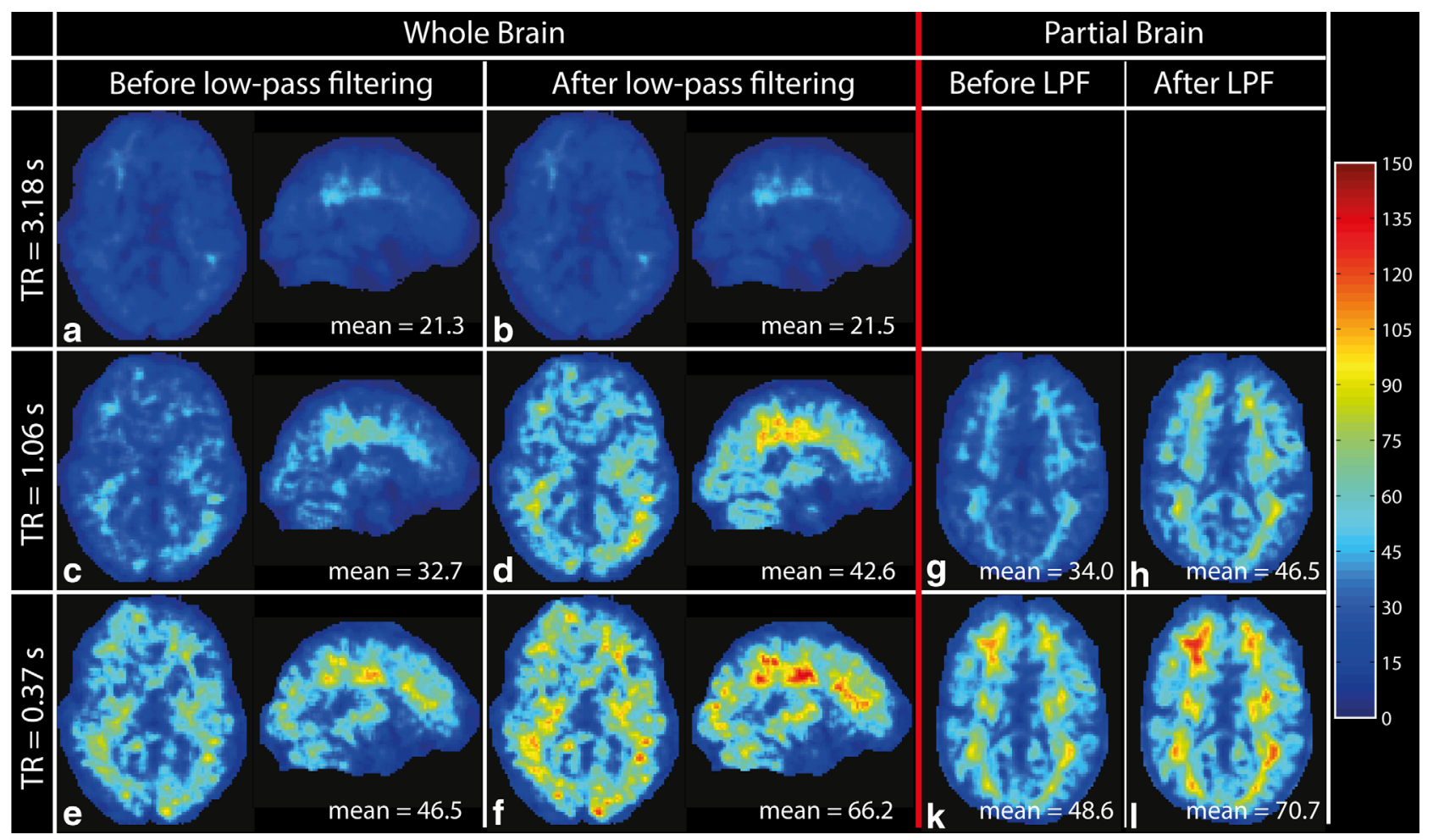

FIG. 4. A-F: Transverse and sagittal view of tSNR per unit scan time maps for TR volume of $3.18 \mathrm{~s}, 1.06 \mathrm{~s}$, and $0.37 \mathrm{~s}$ whole-brain data from three subjects after co-registering to T1 weighted Talairach brain template. Panels A, C, and E show maps before low-pass filtering whereas panels $\mathrm{B}, \mathrm{D}$, and $\mathrm{E}$ show corresponding maps after low-pass filtering the datasets with a cut-off frequency at $0.14 \mathrm{~Hz}$. The mean tSNR per unit scan time values calculated over the whole-brain region are indicated at the bottom-right of each figure panel. G,H,K,L: Similar maps (transverse only) created for partial brain acquisitions where no partial parallel imaging was used but the prescribed numbers of slices were reduced to achieve the same TR as with whole-brain acquisitions.

A stimuli and earlier BOLD peaks in the left motor cortex than both $\mathrm{A}$ and $\mathrm{V}$ stimuli. BOLD responses in the motor cortex, with audiovisual BOLD responses fastest and visual
BOLD responses slowest, matched the pattern observed in reaction times $(\mathrm{AV}<\mathrm{A}<\mathrm{V}$, Fig. 7a) despite the changes in response times only varying by as little as $100 \mathrm{~ms}$.

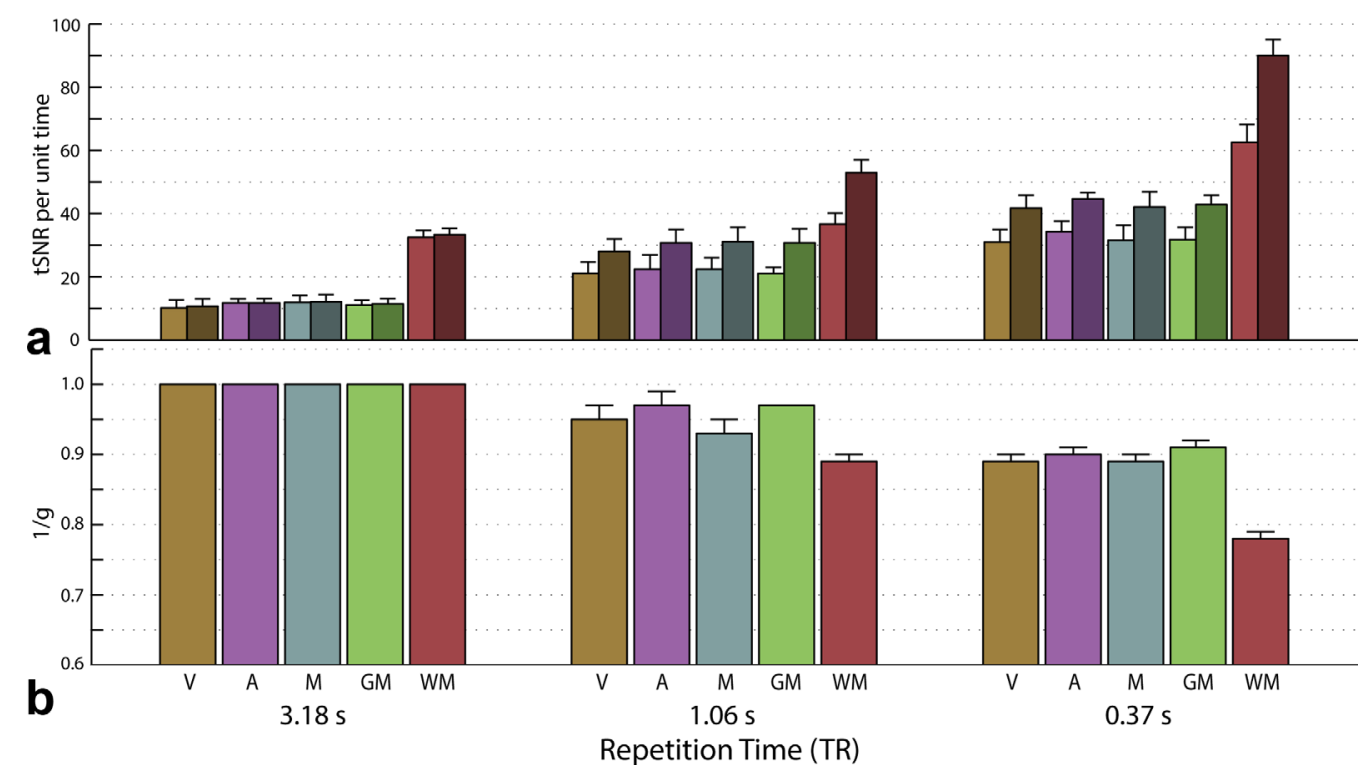

FIG. 5. a: Temporal SNR per unit time values calculated for datasets acquired with $\mathrm{TR}_{\text {volume }}$ of $3.18 \mathrm{~s}, 1.06 \mathrm{~s}$, and $0.37 \mathrm{~s}$. The values were calculated for different brain regions: visual cortex (V), auditory cortex (A), motor cortex (M), whole-brain gray matter (GM), and whole-brain white matter (WM) and averaged across 3 subjects. The values before filtering (light bars) are immediately followed by the values after the application of a low-pass filter (dark bars) with a cut-off frequency $0.14 \mathrm{~Hz}$. b: $1 / \mathrm{g}$ values for the same brain regions averaged over the 3 subjects are plotted for $1.06 \mathrm{~s}$ and $0.37 \mathrm{~s}$ datasets. 


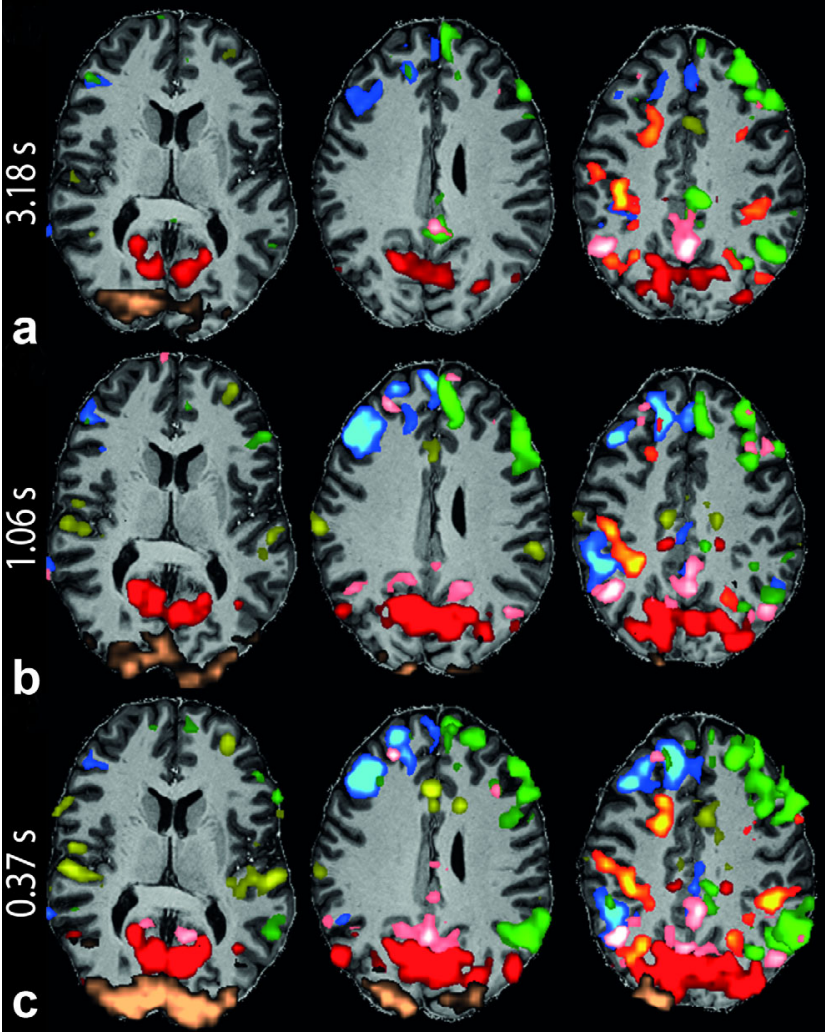

FIG. 6. a-c: Six representative resting state networks identified from datasets acquired with volume TRs of $3.18 \mathrm{~s}, 1.06 \mathrm{~s}$, and $0.37 \mathrm{~s}$. All datasets were low-pass filtered with a cut-off frequency of $0.14 \mathrm{~Hz}$ to filter out respiratory and cardiac signal fluctuations. Furthermore, $1.06 \mathrm{~s}$ and $0.37 \mathrm{~s}$ datasets were temporally downsampled to produce an effective volume TR of $3.18 \mathrm{~s}$ each. All networks were applied a threshold from $\mathrm{z}=5$ to 10 .

\section{DISCUSSION}

The present study demonstrates that CAIPIRINHA acquisition strategies can readily be implemented in 3D-EPI to improve the parallel imaging reconstruction for high acceleration factors. A net acceleration of 10 -fold was shown to be achievable with a 32-channel coil at 7T and was demonstrated to benefit from the CAIPIRINHA sampling pattern. The use of an improved sampling pattern resulted in an increase of average retained SNR of $\sim 10 \%$ and worst-case retained SNR was increased by $\sim 62 \%$ compared with non-CAIPIRINHA acquisition with otherwise the same protocol parameters. This corresponded to a marked improvement in qualitative image quality by means of visual inspection.

To detect activation-induced BOLD responses, reducing the influence of signal fluctuations caused by cardiac pulsation and respiration is especially important at high SNR regime (56), as well as in relatively high resolution data at (ultra) high field $(57,58)$. The effects of heartbeat and breathing are aliased to low frequencies at typical volume repetition times (TRs) of 2-3 s and cannot simply be filtered out. The use of short TRs enables better characterization of these nuisance sources which could then be removed: either by simple low-pass filtering or by means of physiological noise reduction schemes (59-61). Moreover, when using 3D-EPI based sequences, decreasing the number of shots used to form a 3D dataset is expected to improve the tSNR (29) and the temporal acceleration will also reduce the artefacts associated with varying physiology through the volume acquisition. All these effects contribute to the increase of temporal SNR and are not present in 2D-EPI. The application of a low-pass filter to the high sampling-rate $(0.37 \mathrm{~s})$ dataset removed much of the physiological noise, allowing an average increase of tSNR by $42 \%$ in respect to the unfiltered and a close to $208 \%$ with respect to the conventional acquisition (3.18 s). Given that in our resting state experiment (Figure 6 and Table 1) the datasets were down-sampled to the same TR, the improvement in detection of RSNs was dominated by the improved physiological noise removal rather than increased sampling per se.

The detection of subtle changes in HRF shape and dynamics is difficult with data acquired with a volume TR of $2 \mathrm{~s}$ or higher. To obtain sufficient time points to characterize the HRF, many different repetitions can be sampled with slightly differing lags, a technique referred to as "jittering" and also used in by means of (35). Jittering is very sensitive to naturally occurring differences in HRF from event to event and therefore requires a large number of events to be sampled, resulting in long experiments requiring an increased number of participants. Although each timecourse exhibited low noise levels (temporally stable), they had significant differences between their shapes. Sampling each single HRF

Table 1

Names Along with Sizes (in Number of Voxels) and Maximum Z-Score $\left(Z_{\max }\right)$ Values of Resting State Networks Displayed in Fig. 5 with Volume TRs of $3.18 \mathrm{~s}, 1.06 \mathrm{~s}$ and $0.37 \mathrm{~s}^{\mathrm{a}}$

\begin{tabular}{llccc}
\hline Resting state network & Color used & $\begin{array}{c}\mathrm{TR}_{\text {volume }}=3.18 \mathrm{~s} \\
\text { (voxels } / Z_{\text {max }}\end{array}$ & $\begin{array}{c}\mathrm{TR}_{\text {volume }}=1.06 s \\
\left.\text { (voxels } / Z_{\text {max }}\right)\end{array}$ & $\begin{array}{c}\mathrm{TR}_{\text {volume }}=0.371 \mathrm{~s} \\
\left(\text { voxels } / Z_{\text {max }}\right)\end{array}$ \\
\hline Default mode & Pink & $2725 / 15.83$ & $3221 / 16.27$ & $3835 / 19.11$ \\
General visual & Brown & $3432 / 11.86$ & $3968 / 13.10$ & $5634 / 14.92$ \\
Right lateral & Blue & $1871 / 8.58$ & $2629 / 15.47$ & $3792 / 16.79$ \\
Left lateral & Green & $3129 / 12.82$ & $3318 / 14.45$ & $4469 / 14.48$ \\
Medial visual & Red & $3838 / 16.44$ & $4593 / 29.94$ & $5128 / 32.39$ \\
Auditory & Yellow & $829 / 7.45$ & $1545 / 10.59$ & $3749 / 14.83$ \\
Task positive & Red-yellow & $1286 / 12.57$ & $1621 / 13.84$ & $2862 / 15.66$ \\
\hline Mean & - & $2444 / 12.22$ & $2985 / 16.24$ & $4210 / 18.31$ \\
\hline
\end{tabular}

${ }^{a}$ The mean activation map sizes and average of maximum $Z$-scores are also indicated in the last row. 

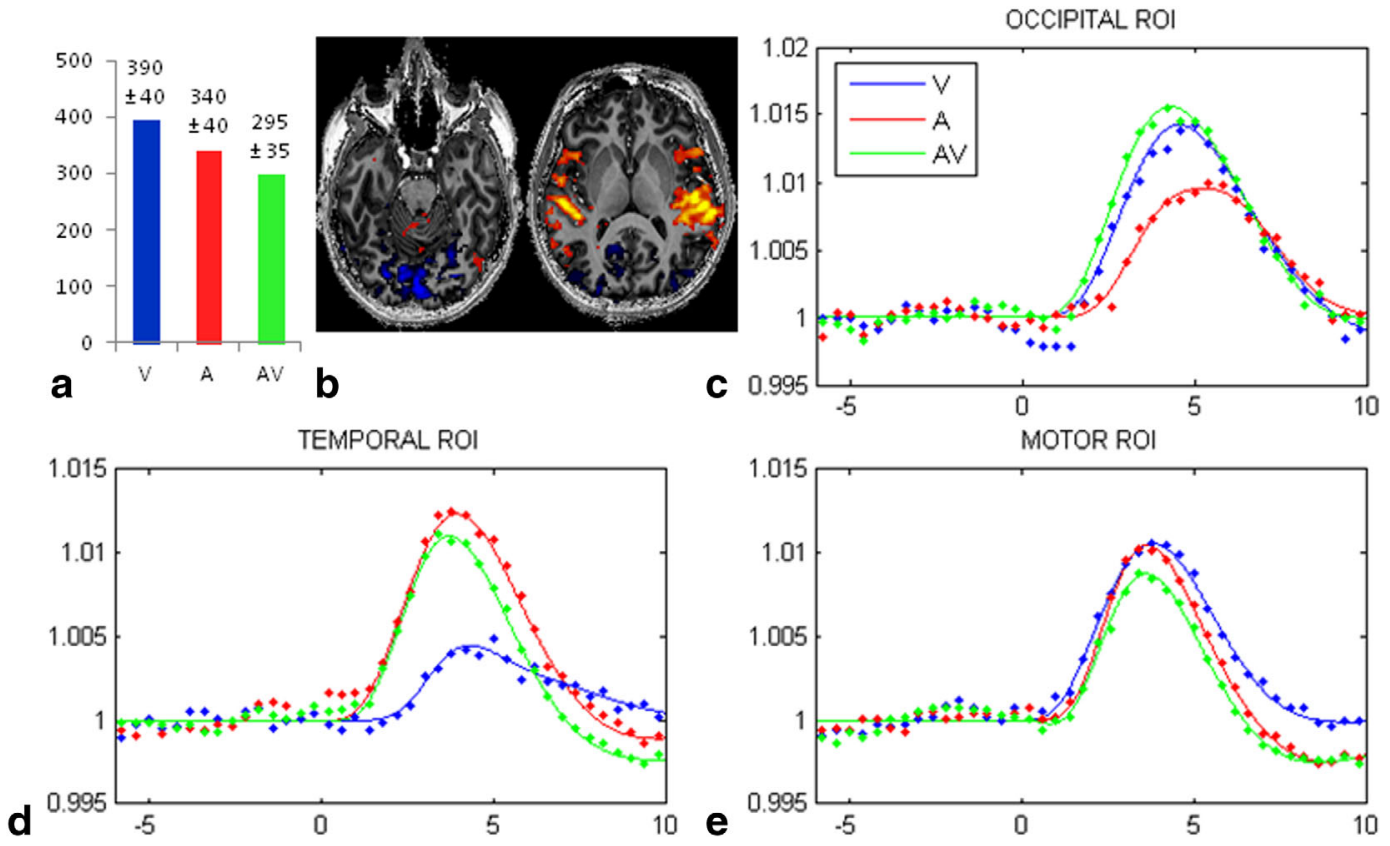

FIG. 7. a: Average reaction times to V, A, and AV stimuli. \pm stderr over runs and volunteers. $\mathbf{b}$ : Example slices from t-score maps (scale 3-10) of a single volunteer for 3D-EPI-CAIPI data. Red/yellow: $A>V$, blue $V>A$. c: 3D-EPI-CAIPI timecourses reordered relative to stimulus onset and fitted double gamma function in the occipital lobe ROI following delivery of $\mathrm{V}$, $\mathrm{A}$, and $\mathrm{AV}$ stimuli timecourses and fitted double gamma function from the temporal lobe ROI (d) and timecourses and fitted double gamma function from the left motor cortex $\mathrm{ROI}(\mathbf{e})$. The dots show the 3D-EPI-CAIPI timecourses; the continuous lines indicate the fitted double gamma functions.

timecourse at sufficient temporal resolution $(<500 \mathrm{~ms})$, reduces this need for averaging, allowing much smaller subject populations to be used. Here, for example, the HRF-differences due to multisensory integration could be observed in a group of 3 volunteers rather than 12, and these 3 subjects only performed two runs each, rather than eight as in the original study (35). In addition, differences in the HRF time-to-peak were also found in the left motor cortex, as might be expected from the differences in reaction times to the differing stimuli, but no such differences could be reported in the original experiment, suggestive of the increased sensitivity of the hightemporal resolution acquisition at higher field strength. Note that these HRF differences were obtained from different brain regions where the vascular compliance, gray matter thickness and venous delay times may differ and these have an important effect on the HRF dynamics. For example, in these data the time-to-peak for the motor cortex is shorter than for the occipital lobe or temporal lobe ROIs, while the neural output of the motor cortex evidently happens after the sensory input is received.

Recently, other groups have started exploring the possibility to use the controlled aliasing in 3D-EPI as a

Table 2

Time to Peak of the Double Gamma Function Fitted to the HRF for Auditory, Visual, and Audiovisual Responses in the Occipital, Temporal, and Left Motor ROls ${ }^{a}$

\begin{tabular}{lccc}
\hline Time to peak (s) & V & A & AV \\
\hline Occipital & 4.70 & 5.42 & 4.46 \\
Temporal & 4.48 & 4.10 & 3.86 \\
Motor & 3.94 & 3.78 & 3.76 \\
\hline
\end{tabular}

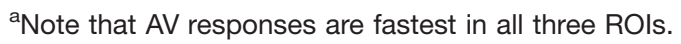

means to obtain higher spatial resolution by translating through-plane sensitivities into in-plane accelerations (61). In our implementation, the use of 3D-EPI-CAIPI is expected to be of greater benefit for fMRI applications where temporal resolution, more than spatial resolution, is a limiting factor. This is especially important when studying rapid transient responses (62) or the shape of the hemodynamic response function as done here in the multisensory integration experiment, or when targeting regions such as the brain stem, where cardiac pulsation plays an important role (63) not only in terms of signal intensity variation but also in terms of motion.

Future work will address a more thorough comparison between 2D-SMS-EPI and 3D-EPI-CAIPI. This comparison has been deemed out of scope for this manuscript because for such a comparison to be fair, many aspects have to be taken into account. For example, similar image reconstruction pipeline for both the sequences (64) and regularization levels for the grappa; similar kspace trajectories; comparison fair Ernst angle comparison and the same degree of fat saturation. The different degrees of sensitivities of the two sequences to physiological signal fluctuations can be assessed through more advanced physiological noise correction techniques such as RETROICOR $(10,59,60)$.

\section{CONCLUSIONS}

We conclude that the standard 3D-EPI sequence can be modified to include CAIPIRINHA-style k-space acquisition patterns, which allow the increase of parallel imaging acceleration in the partition-encoding direction and hence lead to increased tSNR and increased temporal resolution with reduced g-factor penalties compared 
with standard 3D-EPI. With the increased temporal resolution obtained, a better characterization and removal of the physiological noise is possible, thereby improving the ability to detect resting state networks and functional brain activity.

\section{ACKNOWLEDGMENTS}

The authors would like to acknowledge the support of Dr. Felix Breuer for his help with calculation of g-factor maps. The authors also like to acknowledge the support given by Dr. Dominik Nickel and Dr. Tobias Kober from Siemens Healthcare for their help in the online implementation of the CAIPIRINHA reconstruction. Although all functional datasets presented in this manuscript were reconstructed offline to ensure the reconstructions had the reported g-factor properties, the online reconstructions allowed us to quickly assess and ensure the data quality directly at the Siemens console.

\section{REFERENCES}

1. Mansfield P. Multi-planar image formation using NMR spin echoes. J Phys C Solid State Phys 1977;10:L55-L58.

2. Kwong KK, Belliveau JW, Chesler DA, et al. Dynamic magnetic resonance imaging of human brain activity during primary sensory stimulation. Proc Natl Acad Sci U S A 1992;89:5675-5679.

3. Ogawa S, Tank DW, Menon R, Ellermann JM, Kim SG, Merkle H, Ugurbil K. Intrinsic signal changes accompanying sensory stimulation: functional brain mapping with magnetic resonance imaging. Proc Natl Acad Sci U S A 1992;89:5951-5955.

4. Bandettini PA, Wong EC, Hinks RS, Tikofsky RS, Hyde JS. Time course EPI of human brain function during task activation. Magn Reson Med 1992;25:390-397.

5. Norris DG. Principles of magnetic resonance assessment of brain function. J Magn Reson Imaging 2006;23:794-807.

6. Feinberg DA, Moeller S, Smith SM, Auerbach E, Ramanna S, Gunther M, Glasser MF, Miller KL, Ugurbil K, Yacoub E. Multiplexed echo planar imaging for sub-second whole brain FMRI and fast diffusion imaging. PLoS One 2010;5:e15710.

7. Smith SM, Miller KL, Moeller S, et al. Temporally-independent functional modes of spontaneous brain activity. Proc Natl Acad Sci U S A 2012;109:3131-1316.

8. Rabrait C, Ciuciu P, Ribes A, Poupon C, Le Roux P, DehaineLambertz G, Le Bihan D, Lethimonnier F. High temporal resolution functional MRI using parallel echo volumar imaging. J Magn Reson Imaging 2008;27:744-753.

9. Van der Zwaag W, Francis S, Bowtell R. Improved echo volumar imaging (EVI) for functional MRI. Magn Reson Med 2006;56:13201327.

10. Jorge J, Figueiredo P, van der Zwaag W, Marques JP. Signal fluctuations in fMRI data acquired with 2D-EPI and 3D-EPI at 7 Tesla. Magn Reson Imaging 2013;31:212-220.

11. Lutti A, Thomas DL, Hutton C, Weiskopf N. High-resolution functional MRI at 3 T: 3D/2D echo-planar imaging with optimized physiological noise correction. Magn Reson Med 2013;69:1657-1664.

12. Posse S, Ackley E, Mutihac R, Rick J, Shane M, Murray-Krezan C, Zaitsev M, Speck O. Enhancement of temporal resolution and BOLD sensitivity in real-time fMRI using multi-slab echo-volumar imaging. Neuroimage 2012;61:115-130.

13. Setsompop K, Gagoski BA, Polimeni JR, Witzel T, Wedeen VJ, Wald LL. Blipped-controlled aliasing in parallel imaging for simultaneous multislice echo planar imaging with reduced g-factor penalty. Magn Reson Med 2012;67:1210-1224.

14. Nunes RG, Hanjal JV, Golay X, Larkman DJ. Simultaneous slice excitation and reconstruction for single shot EPI. In Proceedings of the 14th Annual Meeting of ISMRM, Seattle, Washington, USA, 2006. Abstract 293.

15. Larkman DJ, Hajnal JV, Herlihy AH, Coutts GA, Young IR, Ehnholm G. Use of multicoil arrays for separation of signal from multiple slices simultaneously excited. J Magn Reson Imaging 2001;13:313-317.
16. Breuer FA, Blaimer M, Heidemann RM, Mueller MF, Griswold MA, Jakob PM. Controlled aliasing in parallel imaging results in higher acceleration (CAIPIRINHA) for multi-slice imaging. Magn Reson Med 2005;53:684-691.

17. Glover GH. Phase-offset multiplanar (POMP) volume imaging: a new technique. J Magn Reson Imaging 1991;1:457-461.

18. Muller S. Multifrequency selective RF pulses for multislice MR imaging. Magn Reson Med 1988;6:364-371.

19. Conolly S, Nishimura D, Macovski A, Glover G. Variable-rate selective excitation. J Magn Reson 1988;78:440-458.

20. Wong EC. Optimized phase schedules for minimizing peak RF power in simultaneous multi-slice RF excitation pulses. In Proceedings of the 20th Annual Scientific Meeting of ISMRM, Melbourne, Victoria, Australia, 2012.

21. Auerbach EJ, Xu J, Yacoub E, Moeller S, Ugurbil K. Multiband accelerated spin-echo echo planar imaging with reduced peak RF power using time-shifted RF pulses. Magn Reson Med 2013;69:1261-1267.

22. Koopmans PJ, Boyacioglu R, Barth M, Norris DG. Whole brain, high resolution spin-echo resting state fMRI using PINS multiplexing at 7 T. Neuroimage 2012;62:1939-1946.

23. Norris DG, Koopmans PJ, Boyacioglu R, Barth M. Power Independent of Number of Slices (PINS) radiofrequency pulses for low-power simultaneous multislice excitation. Magn Reson Med 2011;66:1234-1240.

24. Mansfield P, Coxon R, Hykin J. Echo-volumar imaging (EVI) of the brain at $3.0 \mathrm{~T}$ : first normal volunteer and functional imaging results. J Comput Assist Tomogr 1995;19:847-852.

25. Song AW, Wong EC, Hyde JS. Echo-volume imaging. Magn Reson Med 1994;32:668-671.

26. Abduljalil AM, Aletras AH, Robitaille PM. 3D echo planar imaging: application to the human head. Magn Reson Med 1995;34:144-148.

27. Muftuler LT, Nalcioglu O. Improvement of temporal resolution in fMRI using slice phase encode reordered 3D EPI. Magn Reson Med 2000;44:485-490.

28. Poser BA, Koopmans PJ, Witzel T, Wald LL, Barth M. Three dimensional echo-planar imaging at 7 Tesla. Neuroimage 2010;51:261-266.

29. Van der Zwaag W, Marques JP, Kober T, Glover G, Gruetter R, Krueger G. Temporal SNR characteristics in segmented 3D-EPI at 7T. Magn Reson Med 2012;67:344-352.

30. Narsude M, van der Zwaag W, Kober T, Gruetter R, Marques JP. Improved temporal resolution for functional studies with reduced number of segments with three-dimensional echo planar imaging. Magn Reson Med 2014;72:786-792.

31. Lai S, Glover GH. Three-dimensional spiral fMRI technique: a comparison with 2D spiral acquisition. Magn Reson Med 1998;39:68-78.

32. Hu Y, Glover GH. Three-dimensional spiral technique for highresolution functional MRI. Magn Reson Med 2007;58:947-951.

33. Breuer FA, Blaimer M, Mueller MF, Seiberlich N, Heidemann RM, Griswold MA, Jakob PM. Controlled aliasing in volumetric parallel imaging (2D CAIPIRINHA). Magn Reson Med 2006;55:549-556.

34. Narsude M. MJ, Gallichan D., and Gruetter R. Superior GRAPPA reconstruction with reduced g-factor noise using 2D CAIPIRINHA for 3D EPI. In Proceedings of the 21st Annual Meeting of ISMRM, Salt Lake City, Utah, USA, 2013. Abstract 3705.

35. Martuzzi R, Murray MM, Michel CM, Thiran JP, Maeder PP, Clarke S, Meuli RA. Multisensory interactions within human primary cortices revealed by BOLD dynamics. Cereb Cortex 2007;17:1672-1679.

36. Griswold MA, Jakob PM, Heidemann RM, Nittka M, Jellus V, Wang J, Kiefer B, Haase A. Generalized autocalibrating partially parallel acquisitions (GRAPPA). Magn Reson Med 2002;47:1202-1210.

37. Lin F-H. Prior-regularized GRAPPA reconstruction. In Proceedings of the 14th Scientific Meeting of ISMRM, Seattle, Washington, USA, 2006. Abstract 3656.

38. Qu P, Wang C, Shen GX. Discrepancy-based adaptive regularization for GRAPPA reconstruction. J Magn Reson Imaging 2006;24:248255.

39. Zakhor A. Ghost cancellation algorithms for MRI images. IEEE Trans Med Imaging 1990;9:318-326.

40. Polimeni JR, Bhat H, Witzel T, Benner $\mathrm{T}$, Feiweier $\mathrm{T}$, Inati SJ, Renvall V, Heberlein K, Wald LL. Reducing sensitivity losses due to respiration and motion in accelerated echo planar imaging by reordering the autocalibration data acquisition. Magn Reson Med 2016; 75:665-679.

41. Breuer FA, Kannengiesser SA, Blaimer M, Seiberlich N, Jakob PM, Griswold MA. General formulation for quantitative G-factor calculation in GRAPPA reconstructions. Magn Reson Med 2009;62:739-746. 
42. Eggenschwiler F, Kober T, Magill AW, Gruetter R, Marques JP. SA2RAGE: a new sequence for fast B1+ -mapping. Magn Reson Med 2012; 67:1609-1619.

43. Marques JP, Kober T, Krueger G, van der Zwaag W, Van de Moortele PF, Gruetter R. MP2RAGE, a self bias-field corrected sequence for improved segmentation and T1-mapping at high field. Neuroimage 2010;49:1271-1281.

44. Haacke EM, Lindskogj ED, Lin W. A fast, iterative, partial-fourier technique capable of local phase recovery. J Magn Reson 1991;92: 126-145.

45. Bydder M, Robson MD. Partial fourier partially parallel imaging. Magn Reson Med 2005;53:1393-1401.

46. Jenkinson M, Bannister P, Brady M, Smith S. Improved optimization for the robust and accurate linear registration and motion correction of brain images. Neuroimage 2002;17:825-841.

47. Jenkinson M, Smith S. A global optimisation method for robust affine registration of brain images. Med Image Anal 2001;5:143-156.

48. Greve DN, Fischl B. Accurate and robust brain image alignment using boundary-based registration. Neuroimage 2009;48:63-72.

49. Beckmann CF, DeLuca M, Devlin JT, Smith SM. Investigations into resting-state connectivity using independent component analysis. Philos Trans R Soc Lond B Biol Sci 2005;360:1001-1013.

50. Biswal B, Yetkin FZ, Haughton VM, Hyde JS. Functional connectivity in the motor cortex of resting human brain using echo-planar MRI. Magn Reson Med 1995;34:537-541.

51. De Martino F, Esposito F, van de Moortele PF, Harel N, Formisano E, Goebel R, Ugurbil K, Yacoub E. Whole brain high-resolution functional imaging at ultra high magnetic fields: an application to the analysis of resting state networks. Neuroimage 2011;57:1031-1044.

52. Veer IM, Beckmann CF, van Tol MJ, Ferrarini L, Milles J, Veltman DJ, Aleman A, van Buchem MA, van der Wee NJ, Rombouts SA. Whole brain resting-state analysis reveals decreased functional connectivity in major depression. Front Syst Neurosci [Internet] 2010;4:pii.

53. Shumskaya E, Andriessen TM, Norris DG, Vos PE. Abnormal wholebrain functional networks in homogeneous acute mild traumatic brain injury. Neurology 2012;79:175-82.

54. Glover GH. Deconvolution of impulse response in event-related BOLD fMRI. Neuroimage 1999;9:416-429.

55. Fox MD, Raichle ME. Spontaneous fluctuations in brain activity observed with functional magnetic resonance imaging. Nat Rev Neurosci 2007;8:700-711.
56. Bodurka J, Ye F, Petridou N, Murphy K, Bandettini PA. Mapping the MRI voxel volume in which thermal noise matches physiological noise-implications for fMRI. Neuroimage 2007;34:542-549.

57. Hutton C, Josephs O, Stadler J, Featherstone E, Reid A, Speck O, Bernarding J, Weiskopf N. The impact of physiological noise correction on fMRI at 7 T. Neuroimage 2011;57:101-112.

58. Bianciardi M, van Gelderen P, Duyn JH, Fukunaga M, de Zwart JA. Making the most of fMRI at $7 \mathrm{~T}$ by suppressing spontaneous signal fluctuations. Neuroimage 2009;44:448-454.

59. Bianciardi M, Fukunaga M, van Gelderen P, Horovitz SG, de Zwart JA, Shmueli K, Duyn JH. Sources of functional magnetic resonance imaging signal fluctuations in the human brain at rest: a $7 \mathrm{~T}$ study. Magn Reson Imaging 2009;27:1019-1029.

60. Glover GH, Li TQ, Ress D. Image-based method for retrospective correction of physiological motion effects in fMRI: RETROICOR. Magn Reson Med 2000;44:162-167.

61. Poser BA, Kemper VG, Ivanov D, Kannengiesser SA, Uludag K, Barth M. CAIPIRINHA-accelerated 3D EPI for high temporal and/or spatial resolution EPI acquisitions. In Proceedings of the 30th Annual Scientific Meeting of ESMRMB, Toulouse, France, 2013. Abstract 226.

62. Afacan O, Hoge WS, Janoos F, Brooks DH, Morocz IA. Rapid fullbrain fMRI with an accelerated multi shot 3D EPI sequence using both UNFOLD and GRAPPA. Magn Reson Med 2012;67:1266-1274.

63. Guimaraes AR, Melcher JR, Talavage TM, Baker JR, Ledden P, Rosen BR, Kiang NY, Fullerton BC, Weisskoff RM. Imaging subcortical auditory activity in humans. Hum Brain Mapp 1998;6:33-41.

64. Zahneisen B, Poser BA, Ernst T, Stenger VA. Three-dimensional Fourier encoding of simultaneously excited slices: generalized acquisition and reconstruction framework. Magn Reson Med 2014;71:20712208 .

\section{SUPPORTING INFORMATION}

Additional Supporting Information may be found in the online version of this article.

Figure S1. GRAPPA kernels $(3 \times 3)$ used for image quality comparison (Fig. 2) with different CAIPIRINHA shifts $(\Delta)$. The care was taken to ensure that the same numbers of source points (acquired data) contribute toward estimation of target points (un-acquired/skipped data) for a same overall acceleration factor irrespective of the $\Delta$ used. This would remove any bias in the quality of the images caused due to different source points to target points ratio. 